\title{
The Mediating Role of Professional Commitment in Self-efficacy and Learning Burnout of Special Education Majors - Take China's Southwestern Part as an Example
}

\author{
Yue Liu \\ College of Education, Beijing Sport University, Beijing 100084, China \\ Email: tjliuyue66@163.com
}

Abstract: Through the questionnaire for the professional commitment, self-efficacy, academic burnout of the southwestern part, and explore the relationship between the three and its intrinsic mechanisms, in order to reduce learning burnout of normal students in special education, improve their Academic quality provides effective recommendations. The results showed that self-efficacy positively affected the low sense of achievement in learning burnout, negatively affected the inappropriate behavior, and had no significant effect on the depression. Among them, self-efficacy positively affects professional commitment, and then significantly negatively affects low sense of achievement in learning burnout. Professional commitment has a complete mediating effect between self-efficacy and low sense of achievement, while professional commitment has no mediating effect between self-efficacy, low mood and inappropriate behavior.

Keywords: special education, students, professional commitment, learning burnout, self efficacy, mediating effect

\section{Introduction}

In the past few decades, the quality of China's higher education has a huge development in quantity or scale, but there is still a big gap with developed countries compared to developed countries. With the arrival of the information age, the state's requirements for education have become higher and higher. Special education has also developed, in this context, 2012 , "Opinions on Strengthening the Construction of Special Education Teachers" were approved by the Ministry of Education ${ }^{[1]}$, the opinion made the first time for special teachers' construction for the first time, this It fully illustrates the concern and pay attention to special education. The cultivation of special education teachers and students is a key step in the construction of teachers in China. It determines the quality of special education teachers. However, the current situation is not optimistic. It is understood that there have been less than $50 \%$ of professional training teachers in the post-employed professional teachers, which seriously affects the development of special education ${ }^{[2]}$. In addition, the competition in the 21 st century is the competition of talents. The pressure of higher education students has gradually increased, and special education teachers are not exception. The resulting pressure, academic pressure, etc. have caused learning burnout, and seriously hinders special education. The quality of teachers' students. Therefore, what should the quality of special education teachers should improve, how is the learning burnout of special education students, how is the self-efficacy of professional identity, how is the selfefficacy in learning? These are all worthy of our concern and pay attention.

Learning burnout and professional commitment is two important indicators to reflect students' learning psychology ${ }^{[3]}$. Learning burnout is from professional burnout ${ }^{[4]}$, there are currently a variety of learning burnout definitions. Generally speaking, negative attitudes and behaviors that are tired of learning due to study pressure or lack of learning interest ${ }^{[5]}$. When students produce burnout, there will be some psychological and physiological behavior of tired, fatigue ${ }^{[6]}$. In recent years, learning is getting more and more attention ${ }^{[7-8]}$. In the study of learning burnout to Teaching Teachers, some scholars have found that the overall situation of the special teacher Fan Sheng learning is not optimistic. In the object of its survey, nearly $30 \%$ of students showed burnout, and There are $60 \%$ of uncertain, once there is no good education and guidance will also enter the learning burn ${ }^{[3]}$, this is worth the attention. Professional commitments have come from organizational commitments and believe that the commitment is consistent mechanism, which is consistent ${ }^{[9]}$. The current relatively general statement believes that professional commitments are positive attitudes and behaviors that college students are recognized by school and willing to pay corresponding efforts ${ }^{[10]}$. In the current learning theory, self-efficacy has become one of the focus of many scholars. The self-efficacy is the first to be defined by the psychologist class Dura. He believes that the selfefficacy is the judgment of the individual's ability to engage in certain unopened activities ${ }^{[1]}$. In the study of the three people, Li Xiaohua ${ }^{[12]}$ believes that self-efficacy is the main predictor of learning burnout, self-efficacy and low achievements in 
learning burnout, and the mood is negative. Liang Xiao ${ }^{[13]}$ is a significant correlation between master's self-efficacy and performance in learning burnout. Chen Jiaxiang ${ }^{[14]}$ believes that professional commitments and self-efficacy can predict learning burnout. There are also foreign research scholars that professional commitments and learning burnout factors (emotional low, misconduct, and low awareness) ${ }^{[15]}$. In addition, Zhao Wei ${ }^{[16]}$ has pointed out that professional commitment has different intermediary roles between college students 'self-efficacy and learning burnout factors, and their studies have shown that college students' self-efficacy and learning burnout. Part of the mediation effect, a complete mediation role between misconduct in the behavior of learning burnout, can't afford the agency between the achievements. The study further studied the role mechanism between the three, but the study object is too extensive, and there is no targeted for special educational teachers. Therefore, it is found that the research of most scholars at home and abroad found that although the researchers found self-efficacy, learning burnout, and the two relationships of the professional commitment, the mechanism of self-efficacy on learning burnout, there are very few people to conduct comprehensive Discussion and verification, more relatively relatively small forr future special education teachers ${ }^{[17]}$.

Special education students' study burnout will bring many adverse effects on their professional development and their own growth. The exploration of the special education majors in the secondary effects of self-efficacy and learning burnout can provide certain inspiration and recommendations to our higher special education construction, which will play an important guiding role in relieving special education college students. In view of this, this paper investigates and analyzes the status of their study burnout, self-efficacy, and professional commitment, and try to explore the professional commitment of special education teachers and students. There may be an intermediary effect between efficacy and learning burnout, in order to provide some assistance to the quality of students with special education majors.

\section{Research methods}

\subsection{Research object}

This study selected the first year, second grade, third grade and fourth grade students of special education majors in southwestern China. The basic situation of the test is shown in Table 1 and Table 2.

Table 1. Research of the proportion of men and women

\begin{tabular}{ccc}
\hline Sex & Number & Ratio \\
\hline Male & 15 & $10.14 \%$ \\
Female & 133 & $89.86 \%$ \\
\hline & Table 2. Grade ratio of research subjects & \\
\hline Grade & Number & Ratio \\
\hline Freshman & 19 & $12.84 \%$ \\
Sophomore & 36 & $24.32 \%$ \\
Junior year & 36 & $24.32 \%$ \\
Senior year & 57 & $38.51 \%$ \\
\hline
\end{tabular}

\subsection{Research design}

\subsubsection{Research purposes}

Special education majors have different students with ordinary education majors, and their psychological features are different. This study selected four grades of special education majors in southwestern China as a research object for questionnaire survey. Through the "Study Turning Survey Survey Meter of College Students", "College Students 'Professional Commitment Survey Table", "College Students' Learning Self-Volume Strike Survey Meter" (GSES) Three Survey Table "The three survey measures of special education teachers are committed to learning tired and self-efficacy The relationship between the senses and discusses whether there is an intermediary effect, revealing the inherent mechanism of the three, in order to enhance the professional identity and self-efficacy level of special education teachers, effectively alleviate its learning burnout, and improve the quality of Teaching talents.

\subsubsection{Research assumption}

Based on the preliminary literature review and the following assumptions on the basis of predecessors. 
Research hypothesis 1: Special education teachers' self-efficacy, professional commitment and learning burnout factor (emotional low, behavioral, and low awareness) have different degrees of impact. According to the predecessors' research on the three and the perception of the three, it will be proposed in turn.

Sub assumption 1: Special Education Normal School Students Self - efficacy Negative Emotional Emotions in Learning Burnout;

Sub assumption 2: The self-efficacy of special education teachers' self-efficacy is not active in learning burnout;

Sub assumption 3: Special Education Teachers' Self - efficacy Negative Negative Affecting Affects Learning in Learning Burnout.

Research hypothesis 2: Special education teachers' professional commitment and self-efficacy have positive relationships, the higher the self-efficacy of students, the higher the professional commitment, the higher the level.

Research assumptions 3: Special education teachers' professional commitment may have an intermediary role between self-efficacy and learning burnout factors (emotional low, misconduct, and low awareness). Specifically, it can be divided into three subsistences.

Sub assumption 4: Teacher's professional commitments have an intermediary role in self-efficacy and emotional low;

Sub assumption 5: Professional Commitment of Teaching Teachers Colleges in the Self - efficacy and misconduct;

Sub assumption 6: Special Education Teachers' Professional Commitment has an intermediary role in self-efficacy and achievements;

\subsubsection{Research framework}

According to the above, the research framework of this paper is shown in Figure 1:

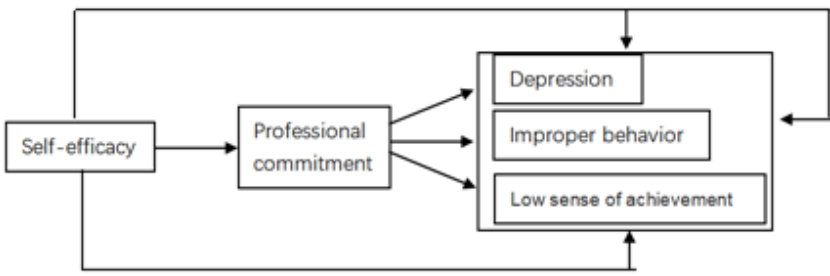

Figure 1. Research framework model

\subsubsection{Intermediary effect analysis}

Intermediary Effect Analysis According to Wen Zhonglin proposed intermediary effect effect inspection procedures and methods ${ }^{[18]}$. The self-variable $(\mathrm{X})$ in the study is self-efficient, and the variable $(\mathrm{Y})$ is three factors in learning burnout, the intermediary variable $(\mathrm{m})$ is a professional commitment. Where the interpolation path diagram is shown in Figure 2, the specific steps are as follows.

Step 1: $\mathrm{X}$ is regained for $\mathrm{Y}$, the verification of the variable is remarkable due to the variable, that is, whether the $\mathrm{c}$ is significant in equation (1), if it does not have intermediary effect, the test procedure is closed. If significant, the second step is performed.

Step 2: X to M Make regression inspection, check whether the argument is remarkable to the intermediary variable, that is, whether the A in equation (2) is significant, if there is no intermediary effect, stop the intermediary effect test, if it is remarkable. Next step.

Step 3: $\mathrm{X}$ and $\mathrm{M}$ are registered with $\mathrm{Y}$. If the coefficient $\mathrm{b}$ is significant, there is an intermediary effect. If $\mathrm{c}$, it is a partial intermediary, which is not significant is a complete intermediary.

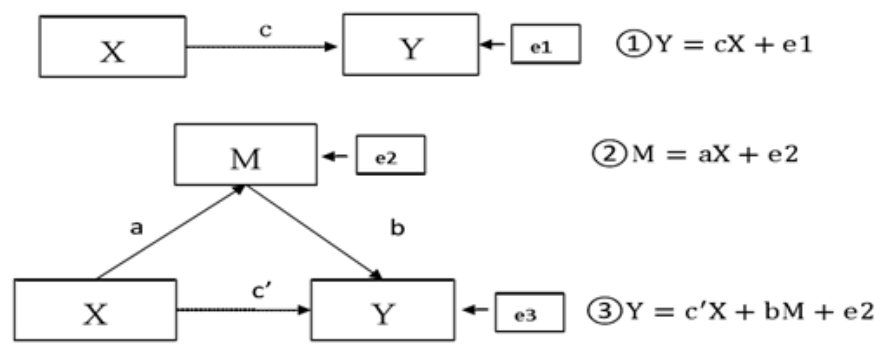

Figure 2. Medium detection path 


\subsection{Research tools}

(1) Investigation scale of College Students' Learning Burnout.

The scale was compiled by Lian Rong and others in 2005 according to Maslach (1997). It includes three dimensions: depression, improper behavior and low sense of achievement, with a total of 20 questions ${ }^{[15]}$. Cronbach of the total questionnaire $\alpha$ The coefficient was 0.865 and the split half reliability was 0.880 . The correlation between different items and the total questionnaire was 0.408-0.762 [16].

(2) Survey scale of College Students' professional commitment.

The measurement of professional commitment is the survey scale of College Students' professional commitment, which was compiled by Lian Rong and others in 2005 with reference to the scale of Meyer and Allen (1993), with a total of 27 questions. Among them, the cronbacha coefficient of the total questionnaire is 0.927 and the split half reliability is 0.831 . The correlation between each item and the total scale is $0.360-0.873$, and the correlation between the four subscales and the total scale is $0.873,0.891,0.774$ and 0.781 respectively; The indicators of confirmatory factor analysis ranged from 0.93 to 0.99 .

(3) College Students' learning self-efficacy survey scale (GSEs).

The scale was compiled by Schwarzer et al. And Wang caikang et al. (2001) revised the Chinese version with reference to the scale. There are 10 questions in the scale, and each question has 1-4 points. The higher the score, the stronger the sense of self-efficacy ${ }^{[19]}$. The cronbacha coefficient of the questionnaire was 0.87 , the split half reliability was 0.82 , and the test-retest reliability was 0.83 .

\subsection{Research process}

1. The survey questionnaire is collected by the combination of electronic questionnaire and collective survey. The purpose and answer method of the electronic questionnaire. For collective testing, the tester shall inform the respondents of the purpose, filling method and other related matters, and the subjects shall answer independently according to the prompts.

2. Questionnaire screening this study adopts the questionnaire survey method. A total of 150 questionnaires are distributed, two invalid questionnaires are excluded, and 148 questionnaires are recovered. The recovery effective rate of the questionnaire is $98.67 \%$.

3. Data analysis and processing.

4. Draw research conclusions and write research reports.

\subsection{Data analysis}

After the questionnaire was collected, the data were entered into SPSS 22.0, the data were processed according to the research purpose and research hypothesis, and the mediation effect was tested by the mediation effect test procedures and methods proposed by Wen Zhonglin.

\section{Research results}

Learning burnout is divided into three dimensions. In order to better understand the causes of learning burnout of normal students in special education and put forward more targeted suggestions, this study tests the three dimensions of learning burnout in turn. Firstly, it tests the depression in learning burnout, tests the relationship between self-efficacy and depression, and whether professional commitment plays an intermediary role. Secondly, it tests the relationship between self-efficacy and misconduct, and whether professional commitment plays an intermediary role. Finally, it tests the low sense of achievement in learning burnout, tests the relationship between self-efficacy and low sense of achievement, and whether professional commitment plays an intermediary role. SPSS 22.0 is used as the test tool for three dimensions in this study, and the test results are as follows.

Table 3. Test of mediating effect of professional commitment on self-efficacy and depression

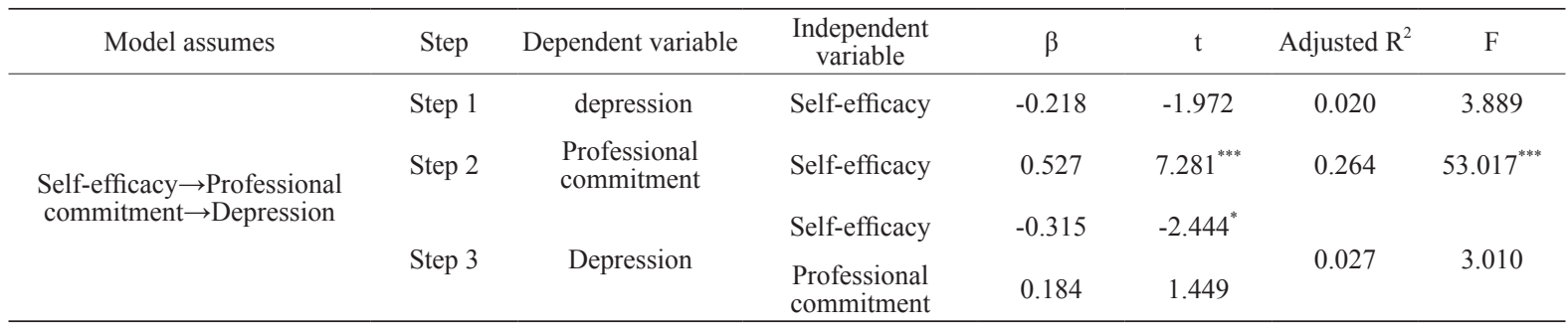

Note: ${ }^{* *} \mathrm{P}<0.05,{ }^{* * * *} \mathrm{P}<0.01$

It can be seen from Table 3 that taking self-efficacy as the independent variable, professional commitment as the 
intermediary variable and depression as the dependent variable for regression analysis, in the first step, the self-efficacy of the independent variable has no significant impact on the depression of the dependent variable $(\mathrm{P}>0.05)$, and the test procedure ends in the first step, so the intermediary effect is not significant. Sub hypothesis 1 and sub hypothesis 4 are not tenable.

Table 4. Test of mediating effect of professional commitment on self-efficacy and improper behavior

\begin{tabular}{|c|c|c|c|c|c|c|c|}
\hline Model assumes & Step & Dependent variable & $\begin{array}{l}\text { Independent } \\
\text { variable }\end{array}$ & $\beta$ & $\mathrm{t}$ & Adjusted $\mathrm{R}^{2}$ & $\mathrm{~F}$ \\
\hline \multirow{4}{*}{$\begin{array}{c}\text { Self-efficacy } \rightarrow \text { Professional } \\
\text { commitment } \rightarrow \text { Improper } \\
\text { behavior }\end{array}$} & Step 1 & Improper behavior & $\begin{array}{l}\text { Professional } \\
\text { commitment }\end{array}$ & -0.246 & $-2.815^{* *}$ & 0.046 & $7.924^{* * *}$ \\
\hline & Step 2 & $\begin{array}{l}\text { Professional } \\
\text { commitment }\end{array}$ & Self-efficacy & 0.527 & $7.281^{* * * *}$ & 0.264 & $53.017^{* * * *}$ \\
\hline & & & Self-efficacy & -0.315 & $-3.088^{* *}$ & \multirow[b]{2}{*}{0.050} & \multirow[b]{2}{*}{$4.829^{* *}$} \\
\hline & Step 3 & Improper behavior & $\begin{array}{l}\text { Professional } \\
\text { commitment }\end{array}$ & 0.131 & 1.302 & & \\
\hline
\end{tabular}

Note: ${ }^{* *} \mathrm{P}<0.05,{ }^{* * *} \mathrm{P}<0.001$

It can be seen from Table 4 that the regression analysis is carried out with self-efficacy as the independent variable, professional commitment as the intermediary variable and misconduct as the dependent variable. In the first step, the selfefficacy of the independent variable has a significant negative impact on the misconduct of the dependent variable ( $\beta=$ $-0.246, \mathrm{P}>0.05$ ). In the second step, the independent variable self-efficacy has a significant positive impact on the mediating variable professional commitment $(\beta=0.527, \mathrm{P}<0.05)$. In the third step, the intermediary variable is added. The independent variable self-efficacy has a significant negative impact on the dependent variable misconduct $(\beta=-0.315, P<0.05)$, but the mediating variable professional commitment has no significant impact on the dependent variable misconduct $(\beta=0.131, \mathrm{P}$ $>0.05$ ), so the mediating effect was not significant. Sub hypothesis 2 is true and sub hypothesis 5 is not true.

Table 5. Test of mediating effect of professional commitment on self-efficacy and low sense of achievement

\begin{tabular}{|c|c|c|c|c|c|c|c|}
\hline Model assumes & Step & Dependent variable & $\begin{array}{c}\text { Independent } \\
\text { variable }\end{array}$ & $\beta$ & $\mathrm{t}$ & Adjusted $\mathrm{R}^{2}$ & $\mathrm{~F}$ \\
\hline \multirow{4}{*}{$\begin{array}{c}\text { self-efficacy } \rightarrow \text { Professional } \\
\text { commitment } \rightarrow \text { Low sense of } \\
\text { achievement }\end{array}$} & Step 1 & $\begin{array}{l}\text { Low sense of } \\
\text { achievement }\end{array}$ & self-efficacy & 0.220 & $3.381^{* *}$ & 0.067 & $11.470^{* * *}$ \\
\hline & Step 2 & $\begin{array}{l}\text { Professional } \\
\text { commitment }\end{array}$ & self-efficacy & 0.527 & $7.281^{* * * *}$ & 0.264 & $53.017^{* * * *}$ \\
\hline & \multirow[b]{2}{*}{ Step 3} & \multirow{2}{*}{$\begin{array}{l}\text { Low sense of } \\
\text { achievement }\end{array}$} & self-efficacy & 0.011 & 0.166 & \multirow[b]{2}{*}{0.244} & \multirow[b]{2}{*}{$24.343^{* * *}$} \\
\hline & & & $\begin{array}{l}\text { Professional } \\
\text { commitment }\end{array}$ & 0.397 & $5.877^{* * * *}$ & & \\
\hline
\end{tabular}

Note: ${ }^{* *} \mathrm{P}<0.05,{ }^{* * * *} \mathrm{P}<0.01$

It can be seen from table 5 that the regression analysis is carried out with self-efficacy as the independent variable, professional commitment as the intermediary variable and low sense of achievement as the dependent variable. In the first step, the self-efficacy of the independent variable has a significant positive impact on the low sense of achievement of the dependent variable $(\beta=0.220, \mathrm{P}<0.05)$. In the second step, the independent variable self-efficacy had a significant positive impact on the mediating variable professional commitment $(\beta=0.527, \mathrm{P}<0.05)$. In the third step, the intermediary variable was added. The self-efficacy of the independent variable had no significant effect on the low sense of achievement of the dependent variable $(\beta=0.011, \mathrm{P}>0.05)$, the mediating variable professional commitment has a significant positive impact on the dependent variable low sense of achievement $(\beta=0.397, \mathrm{P}<0.05)$, the positive correlation between independent variable self-efficacy and dependent variable decreased, indicating that professional commitment plays a complete intermediary role in low self-efficacy and low sense of achievement. Research hypothesis 2 is true, sub hypothesis 3 is not true, and sub hypothesis 6 is true.

\section{Discussion}

\subsection{The relationship between self-efficacy and depression in learning burnout of normal students in special education}

Relevant studies have found that the formation and change of self-efficacy are affected by four information sources, which respectively transmit certain efficacy information and affect people's efficacy level ${ }^{[20]}$. These four information sources include: direct experience, such as behavior success or failure experience; Alternative experience, that is, the experience gained by watching the success or failure of others; Verbal persuasion refers to a method of changing self-efficacy through the advice, guidance and persuasion of others; Other factors, such as personal psychological state. The results of this study 
show that the self-efficacy of normal students in special education has no significant impact on the depression of learning burnout. The results of this study are different from those of Li Xiaohua ${ }^{[12]}$ and Zhao Jun ${ }^{[16]}$. In addition to the possible impact of the sample size of this study on this result, there are two possible reasons why sub hypothesis 1 is not tenable, The first point is that there are many factors affecting self-efficacy, and personal emotional changes also come from many factors, that is, the self-efficacy and emotional depression of normal students in special education come from many aspects. However, the emotional depression in learning burnout limits the scope of emotional depression, because the emotional depression in learning burnout mainly refers to the inability to deal with the problems and requirements in learning. It shows emotional characteristics such as burnout, depression and lack of interest. In fact, individuals may improve their sense of self-efficacy in other fields, such as self-confidence and other people's support. In addition, the degree of love of special education normal students for their major and the proportion of their major in daily life will also have an impact on the significant degree. The second possible reason is that normal students in special education are different from other normal students because of their special objects. For most normal students in special education, the curriculum they contact is a new field, and the objects they serve are also separated from the students' daily experience. The self-efficacy obtained by students from the experience accumulated over a long period of time in the past may not be completely affected by the depression in learning burnout, or the impact is not significant.

\subsection{The relationship between self-efficacy and improper behavior in learning burnout of normal students in special education}

The results show that the sense of self-efficacy of special education normal students has a significant negative impact on misconduct in learning burnout, that is, the higher the sense of self-efficacy, the smaller the probability of skipping class, not listening to class, being late, leaving early, not handing in homework and other behavioral characteristics. On the contrary, the lower the sense of self-efficacy, the smaller the probability of skipping class, not listening to class, being late, leaving early The more likely you are not to hand in your homework and other behavioral characteristics. The results are consistent with previous studies on ordinary college students. This is not difficult to explain. People with high self-efficacy generally believe that they can learn well, so the possibility of misconduct may be lower than those with low self-efficacy. This is of great significance for how to reduce the improper behaviors of special education normal students such as skipping classes, being late and leaving early. It also provides a direction for managing the routine of special teachers and students and improving the professional ability of special teachers and students.

In the analysis of whether professional commitment plays an intermediary role between self-efficacy and misconduct, it is found that professional commitment does not play an intermediary role between self-efficacy and misconduct, that is, sub Hypothesis 5 is not tenable, which is different from the research results of Zhao Jun ${ }^{[16]}$. In addition to the particularity of the research object, It may be because self-efficacy is significantly negatively correlated with misconduct, and the significant index is high. It is not necessary to have an impact through the intermediary variable of professional commitment. Selfefficacy can directly affect misconduct, so professional commitment has no intermediary effect between self-efficacy and misconduct, or the intermediary effect of professional commitment is not significant.

\subsection{The relationship between self-efficacy and low sense of achievement in learning burnout of normal students in special education}

It is found that the self-efficacy of special education normal students has a significant positive impact on the low sense of achievement in learning burnout, that is, the higher the self-efficacy of students, the easier it is to have a low sense of achievement in learning. Bandura believes that self-efficacy is an individual's belief in his ability to complete tasks ${ }^{[20]}$. Therefore, the higher the level of self-efficacy, the greater the individual's confidence in successfully completing something. However, the lower the individual's sense of achievement when successfully completing something, because the individual feels that this is a thing that should be successful. On the contrary, the lower the individual's self-efficacy, the more they feel that things will not succeed. When they succeed, they will have a higher sense of achievement. The same is true in the study of normal students in special education. Students with high self-efficacy have a lower sense of achievement in learning than students with low self-efficacy.

It is found that self-efficacy does not directly affect the low sense of achievement in learning burnout, and professional commitment plays a complete intermediary role, that is, self-efficacy affects the low sense of achievement in learning burnout through professional commitment, which verifies sub hypothesis 6. In other words, students with higher self-efficacy have stronger professional commitment, and students with higher professional commitment have lower sense of achievement. If students do not have professional commitment, the level of self-efficacy will not have a significant impact on the low sense of achievement in learning burnout, which verifies the importance of professional commitment from different angles. 


\subsection{The mediating role of professional commitment in self-efficacy and learning burnout of normal students in special education}

The results of comprehensive analysis show that the self-efficacy of special education normal students has a positive impact on professional commitment, and then has a significant negative impact on the low sense of achievement in learning burnout. Professional commitment plays a complete intermediary role between self-efficacy and low sense of achievement. Professional commitment does not mediate between misconduct and depression.

\subsection{Shortcomings and advantages of this study}

In this study, 138 students in Southwest China were selected according to the principle of proximity. The sample size is relatively small, resulting in low general applicability and possible deviation of the research conclusion. Although there are inevitable defects, this study still has its value. Firstly, the particularity and uniqueness of its object make the conclusions and suggestions more targeted and effective. Secondly, it can provide some meaningful references for the research in other regions.

\section{Conclusions and suggestions}

\subsection{Conclusions}

Through the questionnaire survey of some normal students in special education, after rigorous data analysis and discussion, the following conclusions are drawn.

(1) The sense of self-efficacy of special education normal students has a positive impact on the low sense of achievement in learning burnout, a negative impact on misconduct, and has no significant impact on depression.

(2) Self efficacy positively affects professional commitment, and then significantly negatively affects the low sense of achievement in learning burnout. Professional commitment plays a complete intermediary role between self-efficacy and low sense of achievement.

\subsection{Suggestions}

The results show that the study burnout and professional commitment of special education teachers, self-efficacy has different degrees of relationships. It can be found that maintaining a suitable self-efficacy and enhanced professional commitment is an important measures to improve the quality of special education teachers. Based on this, it is proposed to give a few suggestions.

(1) Pay attention to the promotion of special education students.

First, university teachers should conduct ideological education for special education teachers, especially for students who study special education because of college entrance examination. To fundamentally transforming their ideas, promoting special education in the form of opening, lectures, etc., stimulating their enthusiasm for professional learning and special education, enhance their sense of responsibility and mission of special education, so that special education teachers It's easy to teach, long-term teaching. Second, college teachers should also update the teaching concept, implement the status of students 'main teachers, and fully explore the proactive initiative of students' professionalism. In addition, we must strengthen the professional education and practice of special education teachers, professional education and professional practices are conducive to the better understanding of special education teachers, so that they are full of confidence in the future career development and establish professional ideals. Professional commitments from the side. Finally, do a good job in the student's professional plan, establish a mentor system, so that students will see their three years and five years or even longer, from the side to improve professional commitments.

(2) Guide students to maintain proper self-efficacy.

A number of studies have shown that reasonable attribution methods affect the self-efficacy of college students, so teachers should guide the correct home, that is, they cannot completely attribute their extrinsic factors, and they cannot completely return the failure to intrinsic factors, so that students always keep the students A suitable self-efficacy, effectively avoiding the escaping, fatigue, no sense of accomplishment in learning. In addition, teachers should help students find their own specialty and advantage, so that they are constantly improving self-efficacy, guiding students to organize colorful activities, allowing them to have potential development in different fields. Finally, teachers should reasonably praise and criticize students, so that they are in a wins and unhappy, and always maintain a suitable self-efficacy. 


\section{References}

[1] Ministry of Education, Central Office, National Development and Reform Commission, etc. Opinions on strengthening the construction of special education teachers. www.gov.cn/jrzg/2012-12/13/content_2289807.htm2013-04-21.

[2] Gu Dingqian. Seize the opportunity to promote the scientific development of special education teaching staff [J]. Modern Special Education, 2009, (9): 5-8.

[3] Zhang Maolin. Study on the current situation of learning burnout in special education normal students and its relationship with professional commitment [J]. China Special Education, 2013(6).

[4] Maslach C. Job burnout: new directions in research and intervention[J]. Current Directions in Psychological Science, 2003, 12(5): 189-193.

[5] Guo Ying, Zhou Wenjing. A review of studies on learning burnout at home and abroad in recent years[J]. Educational Academic Monthly, 2008, (10): 17-20.

[6] Bi Zengzhong, Huang Xiting. The relationship between achievement motivation, turnover intention and burnout of middle school teachers[J]. Psychological Science, 2005, (2): 54-57.

[7] Yang Huizhen. Research on the factors that affect the learning burnout of asset management students and the effectiveness of computer learning [D]. Doctoral dissertation of National Central University, Taiwan, 1998.

[8] Liu Zaihua, Mao Xiangjun. The influence of learning pressure on the learning burnout of in-service graduate students: the moderating effect of social support[J]. China Special Education, 2013(1): 79-84.

[9] Becker, H.S. Notes on the Concept of Commitment [J]. American Journal of Sociology, 1960, (6): 32-42.

[10] Luo Yali, Liu Yanling, Liu Yunbo. Investigation on the Status Quo of Undergraduates' Professional Commitment[J]. Higher Education Exploration, 2008, (2): 120-123

[11] Bandura A. Self-efficacy. Toward a unifying theory of behavioral change[J]. Psychological, 1977, (3): 191-215.

[12] Li Xiaohua. The correlation research of college students' self-esteem, self-efficacy, attribution style and learning burnout[J]. Journal of Heilongjiang Institute of Education, 2011, 30(10): 103-105.

[13] Liang Xiao, Guo Xiaojun, Ding Lingyun, Li Hua. The relationship between self-efficacy, social support and learning burnout of postgraduates[J]. Chinese Journal of Health Psychology, 2011, 19(07): 852-854.

[14] Chen Jiasheng. Research status and prospects of learning burnout [J]. Chinese Journal of Health Psychology, 2016, 24(06): 939-943.

[15] Lian Rong, Yang Lixian, Wu Lanhua. The status and relationship of professional commitment and learning burnout of college students[J]. Psychological Science, 2006, 29(1): 47.

[16] Zhao Jun. The relationship between college students' self-efficacy and learning burnout: the mediating role of professional commitment[J]. Journal of Inner Mongolia Normal University (Education Science Edition), 2013, $26(7):$ 85-87.

[17] Chen Li, Yang Qiong. Research on the relationship between professional identity, learning burnout and learning efficacy of special education normal students[J]. China Special Education, 2018(01): 39-45.

[18] Wen Zhonglin, Zhang Lei, Hou Jietai, etc. Intermediary effect test procedure and its application[J]. Psychological Bulletin, 2004, 36(5): 614-620.

[19] Schnauzer R, ARISTI B. Optimistic Self-beliefs: Assessment of General Perceived Self-efficacy in Thirteen Cultures[J]. World Psychology, 1997, 31(2): 177.

[20] Wei Yaoyang, Li Luxuan. Factors influencing college students' sense of efficacy and countermeasures[J]. Continuing Education Research, 2009, (8): 84-86. 